\title{
Deletion of FOXL2 by CRISPR promotes cell cycle G0/G1 restriction in KGN cells
}

\author{
BIN TANG $^{1}$, YUJIE ZHANG ${ }^{2}$, WEI ZHANG ${ }^{2}$, YUQING ZHU $^{1}$ and SHAOPENG YUAN ${ }^{3}$ \\ ${ }^{1}$ Department of International Medicine, China Japan Friendship Hospital, Beijing 100029; \\ ${ }^{2}$ Shandong Provincial Key Laboratory of Plastic and Microscopic Repair Technology, \\ Institute of Plastic Surgery, Weifang Medical University, Weifang, Shandong 461042; \\ ${ }^{3}$ Beijing Ruijian Technology Co., Ltd., Beijing 100086, P.R. China
}

Received May 26, 2018; Accepted October 19, 2018

DOI: $10.3892 /$ ijmm.2018.3956

\begin{abstract}
Forkhead box L2 (FOXL2), a member of the forkhead family of transcription factors, is important in eyelid and ovary differentiation. Although the function of FOXL2 in organogenesis has been investigated, the detailed mechanisms by which FOXL2 mediates cellular process remain to be fully elucidated. Few FOXL2-knockout cell lines have been reported, which has limited molecular mechanism investigations. CRISPR is a novel gene editing technique that has been widely used in human genetic diseases. In the present study, FOXL2 was disrupted using clustered regularly interspaced short palindromic repeats (CRISPR), and screening of a stable knockout cell line was performed in human ovarian granulosa KGN cells. Three sites (F404, F425 and F446) around the ATG start codon on the FOXL2 DNA sequence were constructed in a guide RNA lentivirus. Targeting F425 was most efficient, and western blot analysis and DNA sequencing of the resulting cells suggested that both FOXL2 alleles were fully disrupted. In addition, flow cytometry results indicated that the knockout of FOXL 2 restricted cell cycle progression at the G0/G1 phase. In addition, the expression levels of cell cycle mediators cyclin D1 and cyclin-dependent kinase 4 were reduced. These results confirmed that FOXL2 disruption in $\mathrm{KGN}$ cells is associated with the cell cycle attenuation.
\end{abstract}

\section{Introduction}

Foxhead box L2 (FOXL2), a single-exon gene located at 3q23, belongs to the forkhead family of transcription factors, and is

Correspondence to: Dr Yuqing Zhu, Department of International Medicine, China Japan Friendship Hospital, 2 Yinghuayuan East Street, Chaoyang, Beijing 100029, P.R. China

E-mail: emmazhu2005@126.com

Dr Shaopeng Yuan, Beijing Ruijian Technology Co., Ltd., 113, Zhichun Road, Haidian, Beijing 100086, P.R. China

E-mail: arcer_yuan@163.com

Key words: forkhead box L2, clustered regularly interspaced short palindromic repeats, ovarian granulosa cell, cell cycle a regulatory mediator in organogenesis (1). It was the earliest known marker of sexual dimorphism in ovarian differentiation in mammals and may have multiple roles in ovarian somatic cell differentiation and follicle development and/or maintenance (2). In addition, sequence comparison of FOXL2 from several vertebrate species has shown that it is a conserved early regulator of vertebrate ovarian development and that early defects in ovarian formation are likely to contribute to the blepharophimosis ptosis epicanthus-inversus syndrome (BPES) phenotype (3).

FOXL2 is required for commitment to ovary differentiation (4). Several novel insights into ovarian follicle development have arisen from the investigation of relevant knockout mouse models (5), and somatic sexual reprogramming of adult ovaries to testes by FOXL2 ablation has been demonstrated in mice (6). The absence of secondary follicles and oocyte atresia in Fox $12^{\text {lacz }}$ homozygous mutant ovary granulosa cells shows that the Foxl2 gene is essential for granulosa cell differentiation and ovary maintenance (7). In Fox12 lacz homozygous mutant mice, the transition from pseudosquamous to cuboidal granulosa cells in the primary follicle cannot occur, indicating FOXL2 may be a regulator of the granulosa cell tumor differentiation process (8). Therefore, fully understanding the function and regulation of FOXL2 may reveal novel avenues for the treatment of ovarian granulosa cell tumors and female infertility in the future. However, the detailed molecular mechanisms involved remain to be fully elucidated. Therefore, investigation into the expression of FOXL2 in human cells is likely to provide useful information.

FOXL2 has several potential gene targets, including gonadotropin releasing hormone receptor (GnRHR), glycoprotein hormone $\alpha$ subunit, follistatin (FST), follicle-stimulating hormone (FSH)- $\beta$ and cytochrome P450 family (CYP)17A1 (9). In addition, steroidogenic acute regulatory protein (StAR) and CYP19A1, two transcriptional targets relevant to granulosa cell biology, have been reported previously in relation to the role of FOXL2 in a granulosa tumor cell line (10). StAR is a late differentiation marker in granulosa cells in pre-ovulatory follicles and CYP19A1 is the gene that encodes aromatase. StAR catalyzes the translocation of cholesterol from the outer to the inner mitochondrial membrane, allowing it 
to be processed into steroid hormones, whereas aromatase is an enzyme that is also involved in steroidogenesis and the conversion of androgens to estrogens in granulosa cells (10).

Clustered regularly interspaced short palindromic repeats (CRISPR) together with CRISPR-associated (Cas) proteins are an elegant adaptive defense mechanism in bacteria and archaea, which provide acquired resistance to invading viruses and plasmids (11-13). Studies have shown that type II CRISPR systems can create a synthetic single-guide RNA (sgRNA) consisting of a fusion of CRISPR RNA (crRNA) and trans-activating crRNA (tracrRNA), which can direct site-specific DNA cleavage by the Cas9 nuclease (14). CRISPR and Cas proteins recognize site-specific nucleases directly through gRNA to generate a DNA double-strand break (DSB) at a specified locus. DSBs can then be repaired by homologous recombination or error-prone non-homologous end joining, resulting in a mutant carrying deletions or insertions at the cut site, leading to an open reading frame shift (15). Therefore, this system can efficiently induce targeted genetic modifications, and use short RNA to direct the degradation of foreign nucleic acids (15). The CRISPR/Cas system has been adapted as an efficient gene-targeting technology with the potential for multiplexed genome editing (16). Reports have demonstrated that CRISPR/Cas-mediated gene editing allows the simultaneous targeting of multiple genes and has been utilized not only in cultured human cells (17-19) but also in animal model establishment $(20,21)$ and cancer therapy (22-24).

It was hypothesized that FOXL2 can be effectively disrupted using CRISPR, therefore, the aim of the present study was to create a stable FOXL2-knockout cell line in KGN human ovarian granulosa cells. This cell line may then assist with future investigations of FOXL2 and its mechanism of action.

\section{Materials and methods}

Cell culture. The KGN human ovarian granulosa cells were supplied by Professor Yiming Mu of the Chinese PLA General Hospital (Beijing, China) and human embryonic kidney (293T and 293A) cells were obtained from the American Type Culture Collection (Manassas, VA, USA). The KGN cells were incubated in Dulbecco's modified Eagle's medium: Nutrient Mixture F-12 (DMEM/F-12; Hyclone; GE Healthcare Life Sciences, Logan, UT, USA), and the 293T and 293A cells were incubated in DMEM (Hyclone; GE Healthcare Life Sciences) supplemented with $10 \%$ fetal bovine serum (FBS; Hyclone; GE Healthcare Life Sciences) and antibiotics at $37^{\circ} \mathrm{C}$ in a humidified atmosphere (Thermo Fisher Scientific, Inc.; Waltham, MA, USA) with $5 \% \mathrm{CO}_{2}$.

Plasmid construction. Three G (N19) NGG sequences, where $\mathrm{N}$ stands for any $\mathrm{A}, \mathrm{T}, \mathrm{G}$, or $\mathrm{C}$ nucleotide, downstream of the FOXL2 translation start site (ATG) were selected as potential target sites with the assistance of the Optimized CRISPR Design website (http://crispr.mit.edu/). The resulting three target sequences were as follows: F404: GCGCGCCGGCTTTGT CATGATGG; F425: GGCCAGCTACCCCGAGCCCGAGG; and F446: GGACGCGGCGGGGGCCCTGCTGG.
The following primers were then synthesized: F404F, 5'-GAAAGGACGAAACACCGCGCGCCGGCTTTGTCATG AGTTTTAGAGCTAGAAAT-3' and F404R, 5'-ATTTCTAGC TCTAAAACTCATGACAAAGCCGGCGCGCGGTGTTTC GTCCTT TC-3'; F425F, 5'-GAAAGGACGAAACACCG GCCAGCTACCCCGAGCCCGGTTTTAGAGCTAGAAAT-3' and F425R, 5'-ATTTCTAGCTCTAAAACCGGGCTCGGG GTAGCTGGCCGGTGTTTCGTCCTTTC-3'; F446F, 5'-GAA AGGACGAAACACCGGACGCGGCGGGGGCCCTGCGTT TTAGAGCTAGAAAT-3' and F446R, 5'-ATTTCTAGCTC TAAAACGCAGGGCCCCCGCCGCGTCCGGTGTTTCGT CCTTTC-3'; CRIF, 5'-GTATTTCGATTTCTTGGCTTTATA TATCTTGTGGAAAGGACGAAACACCG-3' and CRIR, 5'-GTTGATAACGGACTAGCCTTATTTTAACTTGCTATT TCTAGCTCTAAAAC-3'.

To construct the F404 gRNA lentivirus plasmid, F404F and F404R were denatured and annealed to act as the template for CRIF and CRIR polymerase chain reaction (PCR) amplification. PCR was conducted using the following parameters: An initial denaturation at $94^{\circ} \mathrm{C}$ for $3 \mathrm{~min}$, followed by 20 cycles of amplification $\left(94^{\circ} \mathrm{C}\right.$ for $20 \mathrm{sec}, 53^{\circ} \mathrm{C}$ for $20 \mathrm{sec}$, and $72^{\circ} \mathrm{C}$ for $20 \mathrm{sec}$ ) with a final extension step at $72^{\circ} \mathrm{C}$ for $10 \mathrm{~min}$. The PCR products were then inserted into the pLKO.1 plasmid using Gibson master mix according to the manufacturer's protocol (New England Biolabs, Beverly, MA, USA). The F425 and F446 gRNA plasmids were also constructed in a similar manner. A flag-tagged Cas9 gene was cloned into the pShuttle-CMV plasmid using the BglII and XhoI restriction sites. Following linearizing with PmeI, the pShuttle-CMV-Cas9 was co-transformed with the pAdEasy-1 plasmid into Escherichia coli strain BJ5183.(Stratagene, La Jolla, CA, USA) by electroporation using a Bio-Rad Gene Pulser electroporator (Bio-Rad Laboratories, Inc., Hercules, CA, USA).

Lentivirus and adenovirus packaging. To package the gRNA lentivirus, the gRNA plasmid and the packaging plasmids pMD and SPA were co-transfected into 293T cells. At $48 \mathrm{~h}$ post-transfection, the medium was harvested and used to infect KGN cells. The recombinant Ad plasmid was linearized with PacI and transfected into AD-293 cells (Stratagene) to produce the adenovirus.

Cell genomic DNA extraction and target sequence amplification. The genomic DNA of KGN cells derived from single colony was extracted using a kit (Tiangen Biotech Co., Ltd., Beijing, China) following the manufacturer's protocol. The sequence including the target sites was amplified with F296F and F818R primers and purified with a kit (Tiangen Biotech Co., Ltd.). The resulting PCR product was $523 \mathrm{bp}$. The sequences of the primers were as follows: F296F: 5'-CATCCGCAGTCTCCA GAAGTTTGA-3'; F818R: 5'-AGGCCGGGTCCAGCGTCC AGTAGT-3'.

T7 Endonuclease I (T7 EI) digestion. Prior to T7 EI digestion, the PCR product was denatured, by placing the reaction tube in a 500-ml beaker of $94^{\circ} \mathrm{C}$ water, and annealed, by allowing it to cool to room temperature. Following cooling, the PCR product was digested with $\mathrm{T} 7 \mathrm{EI}$ at $37^{\circ} \mathrm{C}$ for $2 \mathrm{~h}$ and then assayed with agarose gel electrophoresis. 
Reverse transcription-quantitative PCR (RT-qPCR) analysis. Total RNA was extracted and purified with the Takara MiniBEST Universal RNA Extraction kit (Takara Bio, Inc., Tokyo, Japan) according to the manufacturer's protocol. For cDNA synthesis, $2 \mu \mathrm{g}$ of RNA was reverse transcribed with PrimeScript RT reagent kit (Takara Bio, Inc.) according to the manufacturer's protocol. The real-time PCR was standardised using SYBR FAST qPCR mix (Takara Bio, Inc.). The primers were as follows: $\beta$-actin, forward 5'-GTCCCTCACCCTCCC AAAAG-3' and reverse 5'-GCTGCCTCAACACCTCAA CCC-3'; StAR, forward 5'-TGGGCATCCTTAGCAACCA-3' and reverse 5'-GGGACCACTTTACTCATCACTTTGT-3'; and CYP19A, forward 5'-TGAGGATCCCTTTGGACGAA-3' and reverse 5'-AATAACCTTGGATTTTAACCACGATAG-3'. qPCR was conducted using the following parameters: An initial denaturation at $95^{\circ} \mathrm{C}$ for $5 \mathrm{~min}$, followed by 25 cycles of amplification $\left(95^{\circ} \mathrm{C}\right.$ for $30 \mathrm{sec}, 60^{\circ} \mathrm{C}$ for $40 \mathrm{sec}$, and $72^{\circ} \mathrm{C}$ for $1 \mathrm{~min}$ ) with a final extension step at $72^{\circ} \mathrm{C}$ for $10 \mathrm{~min}$. The PCR results were quantified using the Eppendorf realplex 4S RTqPCR system. The qPCR data were analyzed with the comparative $2^{-\Delta \Delta \mathrm{Cq}}$ method (25) using $\beta$-actin to normalize the expression data. Values are presented as changes relative to the KGN wt values, which were set to 1 .

Western blot analysis. The cells were collected and lysed with buffer [50 mM Tris- $\mathrm{HCl}, 150 \mathrm{mM} \mathrm{NaCl}, 1 \%$ NP40, 0.1\% SDS, $1 \mathrm{mM}$ DTT (pH 7.5)] and protease inhibitor cocktail (Roche Diagnostics, Mannheim, Germany) and sonicated briefly. Following centrifugation $(12,000 \mathrm{x} \mathrm{g})$ at $4^{\circ} \mathrm{C}$ for $15 \mathrm{~min}$, the supernatant was collected, and protein concentrations were determined using the BCA protein quantification assay kit (Thermo Fisher Scientific, Inc.). The supernatant was boiled in Laemmli buffer and analyzed by western blot analysis; $40 \mu \mathrm{g}$ of protein homogenates were loaded onto a denaturing $10 \%$ sodium dodecyl sulfate-polyacrylamide gel using a Mini Protean apparatus (Bio-Rad Laboratories, Inc.) and transferred onto polyvinylidene difluoride membranes (Merck KGaA, Darmstadt, Germany). The membrane was blocked with Tris-buffered saline containing 5\% non-fat dry-milk at room temperature for $2 \mathrm{~h}$, then exposed to primary antibodies overnight at $4^{\circ} \mathrm{C}$. Based on the described reactivity, anti-FOXL2 antibody (1:1,000; cat. no. ab188584, Abcam, Cambridge, MA, USA) and anti-flag antibody (1:2,000; cat. no. F2555, Sigma-Aldrich; Merck KGaA) were used for detection of the transfection effectiveness, and anti-StAR antibody (1:1,000; cat. no. sc-166821, Santa Cruz Biotechnology, Inc., Santa Cruz, CA, USA), and anti-CYP19A antibody (1:1,000; cat. no. sc-374176, Santa Cruz Biotechnology, Inc.) were used to evaluate the disruption of FOXL2. Anti-cyclin D1 antibody (1:1,000; cat. no. ab134175, Abcam), anti-cyclin-dependent kinase (CDK)4 antibody (1:1,000; cat. no. ab108357, Abcam), and anti-CDK6 antibody (1:1,000; cat. no. ab124821, Abcam) were used to detect knockout of the cell cycle. Anti- $\beta$-actin antibody (1:5,000; cat. no. ab8227, Abcam) was used as a protein loading control. The secondary antibody (anti-rabbit or anti-mouse peroxidase-conjugated IgG, 1:5,000; cat. nos. ab6721 and ab6789, Abcam) was incubated with the membrane for $1 \mathrm{~h}$ at room temperature. The reaction products were revealed by enhanced chemiluminescence (ECL Plus; Thermo Fisher Scientific, Inc.). The intensities of the digitally detected bands were evaluated by densitometry using Image-Pro Plus software (Version X; Media Cybernetics, Inc., Silver Springs, MD, USA).

Flow cytometric analysis of cell cycle and apoptosis. The cells $\left(1 \times 10^{6}\right)$ were collected following trypsinization and washed twice with PBS. The cells were then stained using the cycle staining kit and Annexin V-FITC/PI apoptosis kit (Multi Sciences Biotech Co., Ltd., Hangzhou, China) according to the manufacturer's protocol, respectively. In the cell cycle assay, $2 \times 10^{5}$ cells for each sample were measured with a Becton Dickinson FACSCalibur flow cytometer (BD Biosciences, Franklin Lakes, NJ, USA) and analyzed with ModFit software version 3.0 (Verity Software House; Topsham, ME, USA). Cell cycle status was analyzed using propidium iodide (PI) as a specific fluorescent dye probe. For the apoptosis assay, $1 \times 10^{5}$ cells were measured and analyzed with CellQuest software version 2.0 (BD Biosciences). Annexin V(+)/PI(-) and Annexin V(+)/PI(+) represent the cells in early apoptosis and late apoptosis/necrosis respectively and the proportion of these cells out of the total number of cells analyzed were determined.

$x$ CELLigence real-time cell proliferation analysis. Cell proliferation experiments were performed using the xCELLigence Real Time Cell Analyzer (RTCA) DP instrument (ACEA Biosciences, San Diego, CA, USA). The cells were seeded in 16-well plates (E-plate 16 ACEA Biosciences, Inc.) at a density $2 \times 10^{3}$ and $4 \times 10^{3}$ cells in $150 \mu \mathrm{l}$ medium per well, and impedance measurements were monitored at regular time intervals. The cell growth curves were automatically recorded with the xCELLigence system in real time. The area of growth covered in an E-plate due to cell adhesion was recorded as the cell index (CI). A high CI indicates more cell adhesion and vice versa. The experiment was allowed to run for 3 days and each experiment was performed in triplicate. Data were analyzed using RTCA software version 1.2.

Cell viability assay by BrdU labelling and Cell Counting Kit-8 (CCK-8) assay. The cellular viabilities of the FOXL2-knockout and wt KGN cells were determined using two methods: BrdU labelling and a CCK- 8 assay. The BrdU labelling assays were performed using the APC-BrdU Flow kit (BD Biosciences, cat. no. 552598) according to the manufacturer's protocol. Briefly, during the final $1 \mathrm{~h}$ of culture, the cells were pulsed with $10 \mu \mathrm{M}$ of BrdU APC and 7-AAD. The stained cells were analyzed on a flow cytometer using a low flow rate. For the CCK- 8 assay, the cells were seeded in 96-well plates at a density of $1 \times 10^{4}$ cells per well and incubated for $24 \mathrm{~h}$. The cells were then treated with different concentrations of FBS $(0,2,5$ and $10 \%)$ for $24 \mathrm{~h}$. Following treatment, CCK-8 (10 $\mu \mathrm{l} /$ well) was added to the wells and the cells were incubated at $37^{\circ} \mathrm{C}$ for $1 \mathrm{~h}$. Subsequently, the OD value was we detected at $450 \mathrm{~nm}$ (A450) using a microplate spectrophotometer.

Statistical analysis. The experimental data are expressed as the mean \pm standard deviation. For statistical analysis, Student's t-test for quantitative data was processed with Excel version 2007 (Microsoft Corporation, Redmond, WA, USA). 
A Translation start

GCGGCCCGGCCGCGGCGCGCCGGCTTTGTCATGATGGCCAGCTACCCCGAGCCCGAGGACGCGGCGGGGGCCCTGCTGGCCCCAGAGACCG

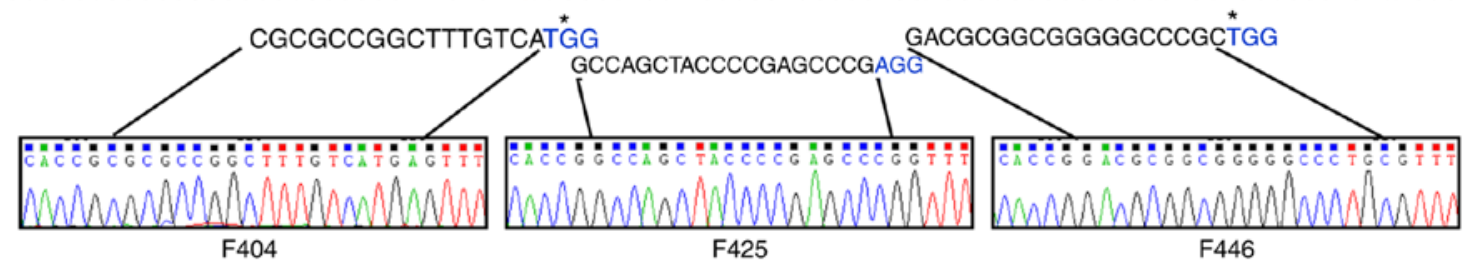

B
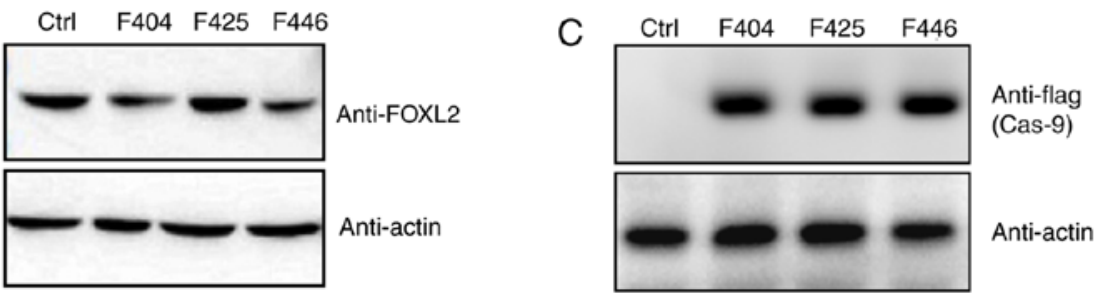

Figure 1. Design and construction of gRNA plasmids and packaging of gRNA lentivirus and flag-Cas9 adenovirus. (A) Three candidate gRNA sequences located downstream of the translation start site were cloned into the pLKO.1 plasmid. (B) gRNA lentivirus was used to infect KGN cells which were then screened with puromycin $(2 \mu \mathrm{g} / \mathrm{ml})$. The expression of FOXL2 was measured with anti-FOXL2 by western blot analysis. (C) Flag-Cas 9 adenovirus was added to KGN cells infected with the respective gRNA lentivirus. The transient expression of flag-Cas9 was measured with anti-flag by western blot analysis. FOXL2, Forkhead box L2; Cas9, CRISPR-associated 9; ctrl, control.

$\mathrm{P}<0.05$ was considered to indicate a statistically significant difference. Error bars represent the standard deviation of at least three independent experiments.

\section{Results}

Knockout of FOXL2 in KGN cells using CRISPR/Cas9. Three potential G (N19) NGG target sites were screened just downstream of the translation start site. All three gRNA sequences were cloned into the pLKO.1 lentivirus vector, and were confirmed by sequencing (Fig. 1A). In addition, as shown in Fig. 1B, gRNA lentivirus infection did not alter the expression of FOXL2. The gRNA lentivirus-infected KGN cells were further infected with flag-Cas9 adenovirus and the expression of Cas9 was monitored by anti-flag western blot analysis (Fig. 1C).

Validation of CRISPR/Cas9-mediated FOXL2-knockout. To assess the efficacy of Cas9-mediated genome editing, the T7 EI digestion of PCR products from the genomic DNA of control and infected KGN cells was analyzed by agarose gel electrophoresis. As shown in Fig. 2A, the PCR products derived from the double infected cells were cut to produce bands of the predicted size, whereas the PCR products of control cells remained almost intact. Quantitative analysis revealed that the cells infected with F425 gRNA were most efficiently edited by Cas9 (Fig. 2B). The luciferase reporter assay results also confirmed that F425 had the highest efficiency of the three targets (Fig. 2C).

A total of $16 \mathrm{~F} 425$ single cell clones were then screened and the genomic DNA of the cells was extracted to perform T7 EI digestion following PCR amplification. As shown in Fig. 3A, the majority of the PCR products from different clones were digested by T7 EI, which included clones 1, 3, 9, 10, 11, 12, 13, 14 , and 16 . The results of the western blot analysis further indicated that clone 3 had been fully disrupted at the FOXL2 locus
(Fig. 3B). To further investigate whether FOXL2 has been completely disrupted in clone 3 , the sequence that included the target sites was cloned into the $\mathrm{T}$ vector and 50 positive clones were sequenced. The sequencing results showed only two genotypes of the target sites, one of which lacked five base pairs and the other lacked four base pairs (Fig. 3C and D).

To further rule out the potential disruption of FOXL2, StAR and CYP-19A, two major reported downstream modulators of FOXL2, were measured. Previous reports have identified that FOXL2 positively regulates StAR and negatively regulates CYP19A (10). As expected, the results showed that StAR was markedly elevated and CYP19A was markedly decreased in clone 3 at the mRNA (Fig. 3E) and protein (Fig. 3F and G) levels. Therefore, the results confirmed that FOXL2 had been successfully knocked out in clone 3 using the CRISPR/Cas9 method.

FOXL2 knockout results in decreased proliferation of $K G N$ cells without affecting apoptosis. To further investigate whether the physiological activities of KGN cells were influenced by FOXL2 knockout, proliferation, cell cycle and apoptosis assays were been performed. The viability assay results using CCK-8 detection showed that, in the presence of different concentrations of FBS $(0,2,5$ and $10 \%)$, the proliferation rate of clone 3 was significantly inhibited, compared with that in the wt cells (Fig. 4A). In addition, the proliferation rate inhibition in clone 3 was confirmed by the xCELLigence RTCA assay (Fig. 4B). However, no significant changes were observed in total apoptosis following the knockout of FOXL2 (Fig. 4C and D). Therefore, the results indicated that FOXL2 knockout mainly attenuated the proliferation of $\mathrm{KCN}$ cells.

FOXL2 knockout restricts cell cycle progression at the G0/GI phase. The present study then measured the effects of FOXL2 knockout on cell cycle. The results showed that the percentage of cells in the G0/G1 phases in clone 3 cells, which lacked 
A
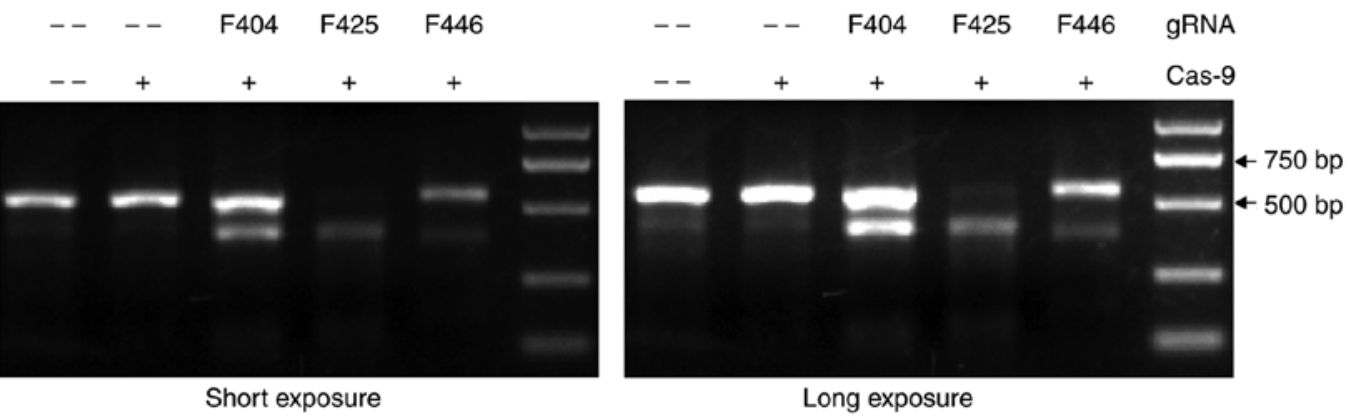

B

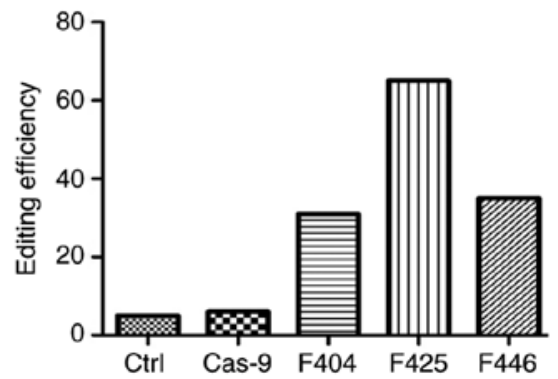

C

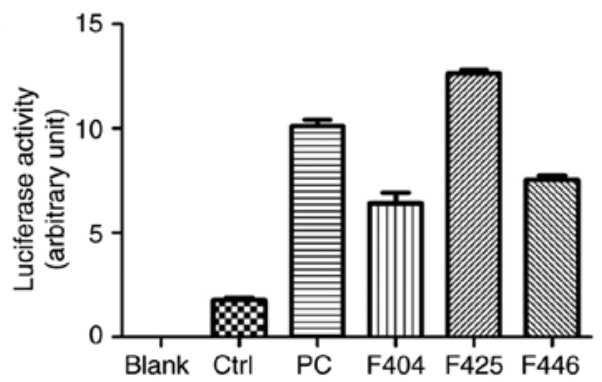

Figure 2. Evaluation of the Cas9 editing efficiency at three gRNA sites. (A) T7 EI digestion assay. Genomic DNA of KGN cells, KGN cells infected with gRNA lentivirus and flag-Cas9 adenovirus were extracted and amplified with PCR. The denatured-annealed PCR products were digested with T7 EI for $1 \mathrm{~h}$ at $37^{\circ} \mathrm{C}$ and then assayed with agarose gel electrophoresis. (B) Bar graph showing quantitative analysis of the T7 EI digestion assay with Quantity One software. Quantitative analysis was performed once. (C) Luciferase reporter assay. The DNA fragment containing the three gRNA target sites were cloned into the pUCA-LUC reporter plasmid. The UCA, gRNA and flag-Cas9 plasmids were co-transfected into $293 \mathrm{~T}$ cells and luciferase activity was measured $48 \mathrm{~h}$ post-transfection. FOXL2, Forkhead box L2; Cas9, CRISPR-associated 9; ctrl, control; PC, positive control; T7 EI, T7 Endonuclease I.

A
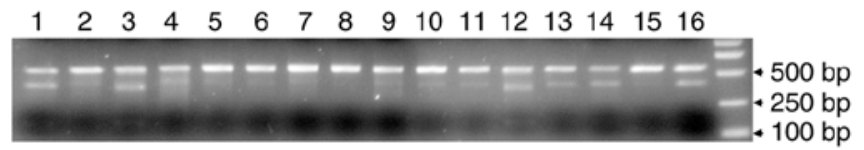

B

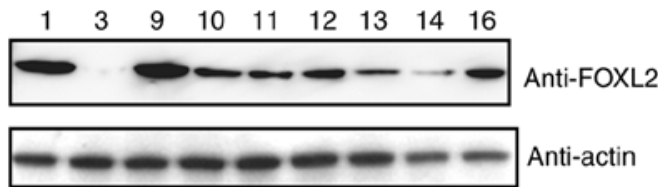

C

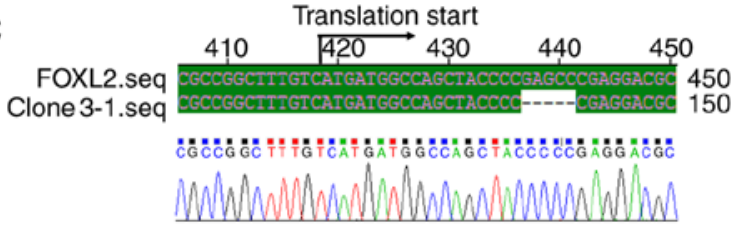

D
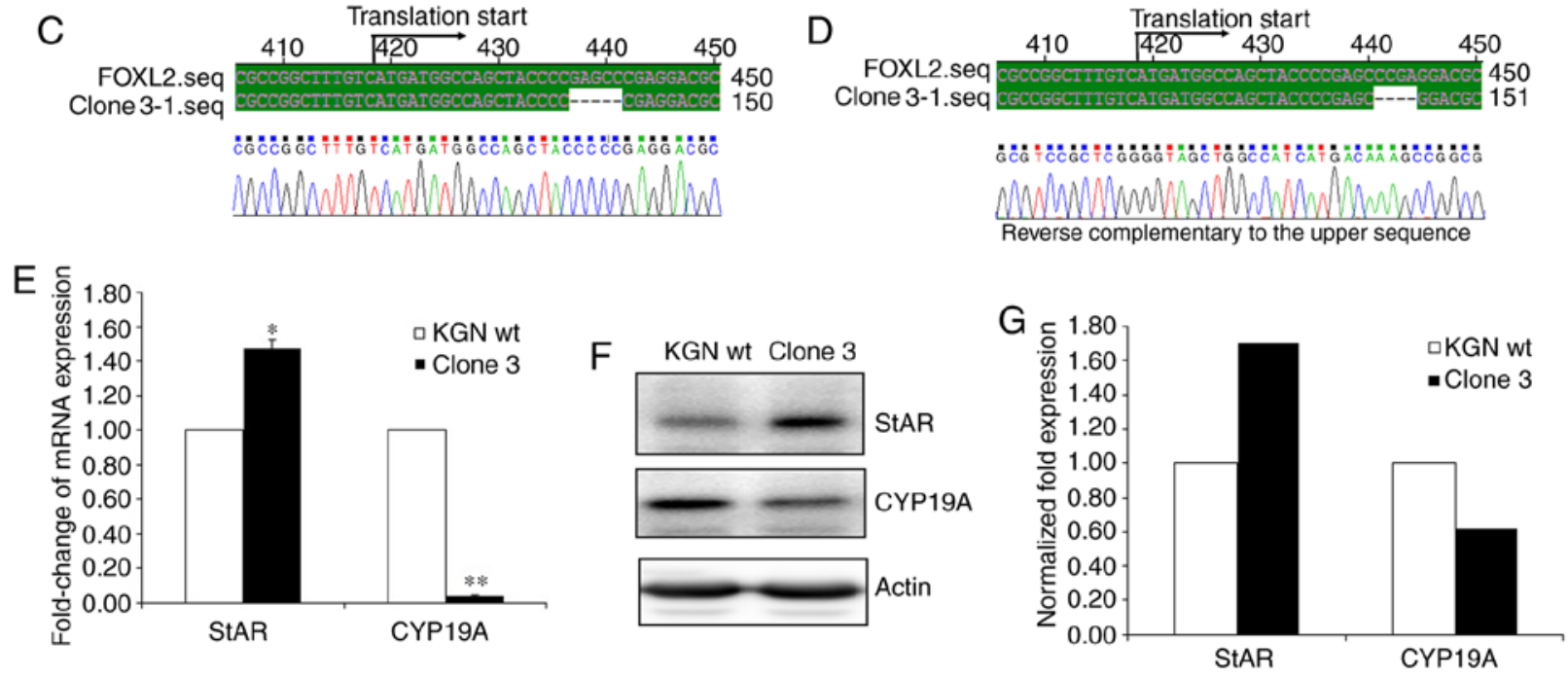

Figure 3. Screening and validation of FOXL2-knockout KCN cells. (A) T7 Endonuclease I digestion screening of KGN single cell clones with mutations in the FOXL2 gene. (B) Western blot screening of FOXL2-knockout KGN single cell clones. (C) DNA sequencing to confirm the FOXL2 disruption revealed a clone with five missing base pairs (D) DNA sequencing to confirm the FOXL2 disruption revealed a clone with four missing base pairs. (E) Reverse transcription-quantitative polymerase chain reaction analysis of StAR and CYP19A in KGN and FOXL2-disrupted cells. P<0.05, ${ }^{* *} \mathrm{P}<0.01$ vs. KGN wt. (F) Western blot analysis of StAR and CYP19A in KGN wt and FOXL2-disrupted cells. (G) Quantitative analysis of the expression of StAR and CYP19A is shown in a bar graph. FOXL2, Forkhead box L2; wt, wild type; StAR, steroidogenic acute regulatory protein; CYP19A, cytochrome P450 family 19A; wt, wild-type.

FOXL2, was significantly enhanced, whereas the percentage in the $\mathrm{S}$ phase was decreased; this suggested that proliferation was inhibited through cell cycle arrest at G0/G1 following
FOXL2 knockout (Fig. 5A and B). The BrdU labelling assays with flow cytometry also confirmed that the clone 3 cells had enhanced G0/G1 phases (Fig. 5C and D). In addition, the results 

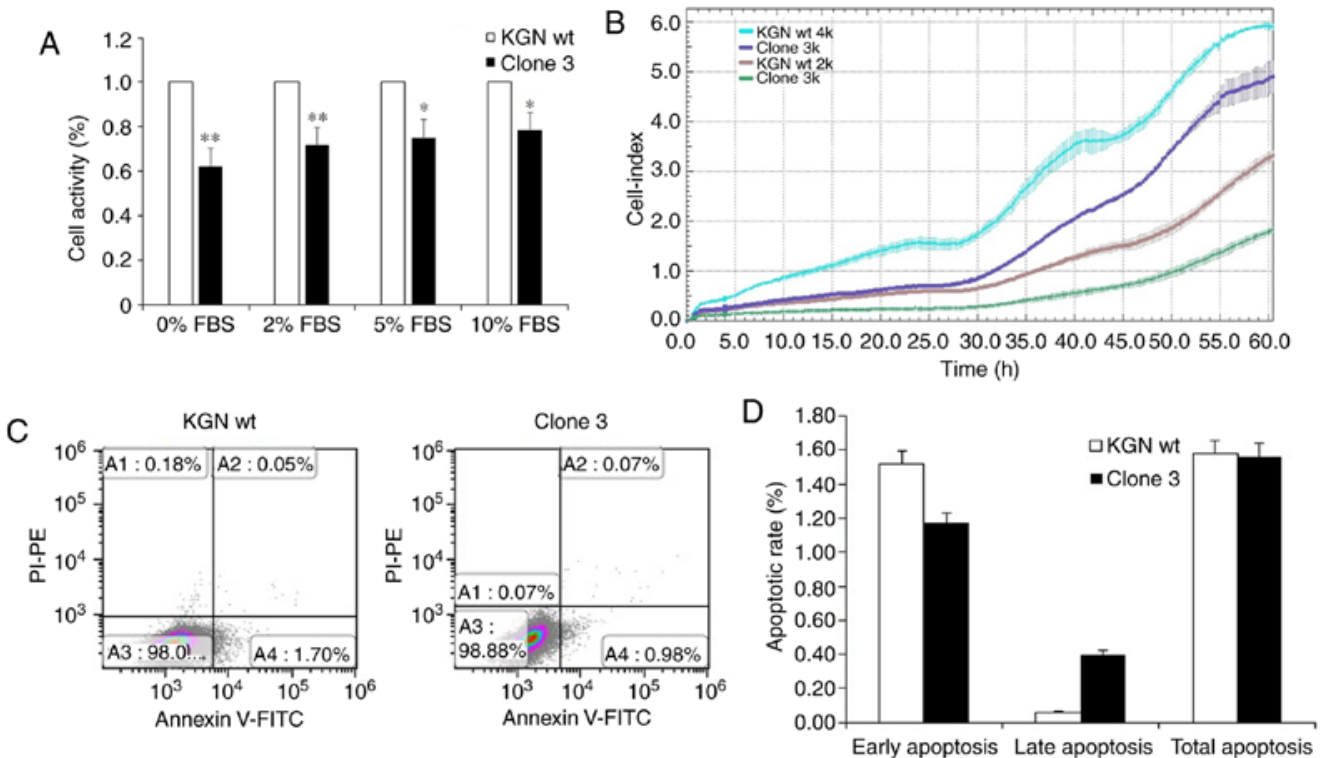

Figure 4. Knockout of FOXL2 in KGN cells results in decreased proliferation. (A) Viable cell number was investigated using the Cell Counting Kit-8, $\mathrm{P}<0.05$, ${ }^{* *} \mathrm{P}<0.01$ vs. KGN wt. (B) Cell proliferation was detected using the $\mathrm{xCELLigence} \mathrm{RTCA} \mathrm{DP} \mathrm{instrument.} \mathrm{Cells} \mathrm{were} \mathrm{seeded} \mathrm{at} \mathrm{a} \mathrm{density} 2 \times 10^{3}$ and $4 \times 10^{3}$ in $150 \mu \mathrm{l}$ medium per well. A high CI indicates increased cell adhesion and vice versa. All data are presented as the mean \pm standard deviation. (C) Cell apoptosis was investigated by flow cytometry using the Annexin V-FITC/PI apoptosis kit. (D) Bar graph showing the apoptotic rate. FITC, fluorescein isothiocyanate; FOXL2, Forkhead box L2; wt, wild-type; FBS, fetal bovine serum; PI, propidium iodide.
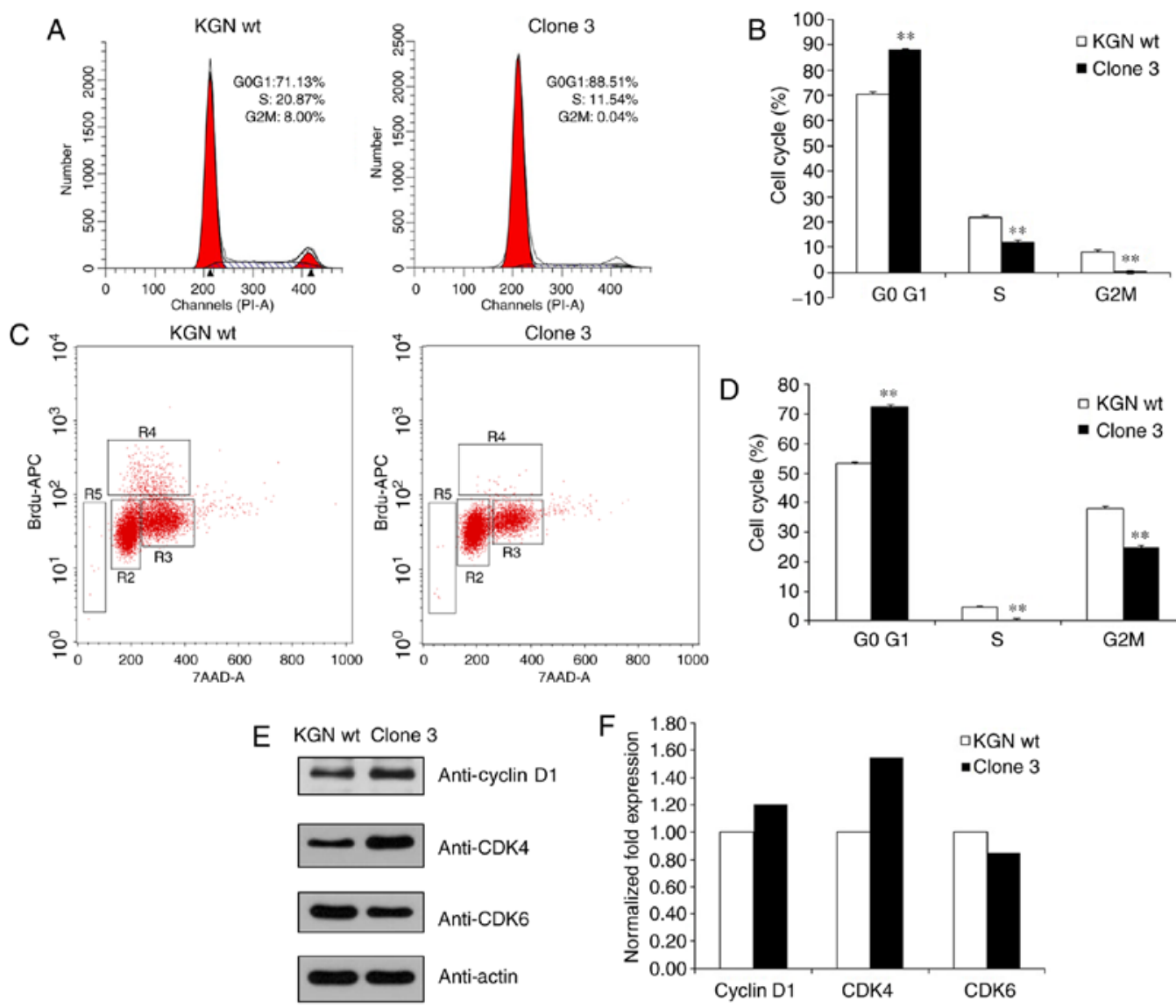

Figure 5. Knockout of FOXL2 attenuates the cell cycle. (A) Analysis of cell cycle distribution of KGN cells with FOXL2 knocked out. (B) Analysis of the cell cycle phases; the bar graph showing the G0/G1, S, and G2/M phases. ${ }^{* *} \mathrm{P}<0.01$ vs. KGN wt. (C) Measurement of cell-incorporated BrdU (with APC anti-BrdU) and total DNA content (with 7-AAD). The cells were cultured with $10 \mu \mathrm{M}$ of BrdU for $1 \mathrm{~h}$. As shown by the regions applied to the 7-AAD, vs. BrdU dot plot, flow cytometric analysis of cells stained with the reagents provided in the BrdU Flow kit allowed for the discrimination of cell subsets that were apoptotic (R5, 0.17 or $0.05 \%$ of cells) or resided in the G0/G1 (R2, 82.79 or $85.73 \%)$, S (R4, 5.17 or $3.50 \%$ ), or G2+M (R3, 10.77 or $9.38 \%$ ) phases of the cell cycle and had recently synthesized DNA. (D) Analysis of the cell cycle phases; the bar graph shows the percentage of cells in the G0/G1, S, and G2/M phases. ${ }^{* *} \mathrm{P}<0.01 \mathrm{vs}$. KGN wt. (E) Protein levels of cyclin D1, CDK4 and CDK6 were determined by western blot analysis. (F) Levels of cyclin D1, CDK4, and CDK6 were quantified using the Quantity One software. Quantitative analysis has been performed once. FOXL2, Forkhead box L2; wt, wild-type; CDK, cyclin-dependent kinase. 
of the western blot analysis demonstrated that the expression of cyclin D1 and CDK4 were upregulated whereas that of CDK6 was downregulated (Fig. 5E and F). This suggested that the knockout of FOXL2 arrested cell cycle progression at the G0/G1 phase. Therefore, the results indicated that knocking out FOXL2 in KGN cells inhibited the proliferation of cells and promoted cell cycle arrest at the G0/G1 phase.

\section{Discussion}

In the present study, FOXL2 was disrupted using the CRISPR method, and stable knockout in the KGN human ovarian granulosa cell line was screened. During the investigations, three sites (F404, F425 and F446) around the ATG start codon on FOXL2 DNA sequence were constructed in gRNA lentivirus. The results showed that F425-targeted gene editing had the highest efficiency among the three candidates. Subsequently, KGN cells infected with F425 gRNA and Cas9 were screened for total FOXL2 knockout. The results of western blot analysis and DNA sequencing identified that one of the screened clones had both FOXL2 alleles fully disrupted. Further investigation indicated that the knockout of FOXL2 inhibited proliferation and restricted cell cycle progression at the G0/G1 phase. Furthermore, the expression levels of cell cycle mediators cyclin D1 and CDK4 were reduced, which confirmed the disruption of FOXL2 in this KGN cell clone, which may be associated with cell cycle attenuation.

The stable cell line with knockout of FOXL2 demonstrated in the present study is likely to provide a useful tool for further investigations into the mechanism of action of FOXL2. FOXL2 is a single-exon gene which belongs to the forkhead transcription factor family (1). The protein sequence of FOXL2 contains a characteristic forkhead box DNA-binding domain and a polyalanine tract of 14 residues of unknown function, which is strictly conserved among eutherian mammals (26). Germline mutations of FOXL2 are responsible for BPES (MIM 110100). Previous studies have also produced models in which the expression of FOXL2 is knocked out. Using a Cre/lox approach, Tran et al (27) selectively ablated FOXL2 in murine anterior pituitary gonadotrope cells and demonstrated that conditional knockout mice developed overtly normally but were subfertile in adulthood. Shi et al (28) disrupted the expression of FOXL2 in mice using piggyBac insertion and successfully mimicked BPES syndrome. Previous investigations in FOXL $2^{-/-}$knockout mice revealed that FOXL2 is critical for ovarian determination and the proper differentiation of granulosa cells. However, the detailed mechanisms by which FOXL2 is able to mediate cellular processes remain to be fully elucidated.

Since the first mammalian applicable CRISPR/Cas9 study was published in 2013 (16), this technique has been successfully used to disrupt several genes in a wide variety of developing organisms (14,29-32). In the present study, FOXL2 was disrupted with CRISPR and a stable knockout cell line was screened in KGN human ovarian granulosa cells. The overall GC content of FOXL2 cDNA is $~ 64 \%$ and, in its coding region, this is $>72 \%$, which limits the gRNA selection due to its high GC content around the ATG site. Fortunately, the free online bioinformatical gRNA design tool (http://crispr.mit.edu/) developed by Hsu et al (33) enabled the selection of three potentially useful gRNA sites in the present study. F425-targeted gene editing exhibited the highest efficiency among the three candidates, and both FOXL2 alleles were fully disrupted in one of the cell clones, as shown in the through western blot analysis and DNA sequencing results. In this clone, the expression of StAR was increased and that of CYP19A was decreased, as expected when FOXL2 is disrupted (10). Therefore, the results reported herein may provide a useful tool to delineate the function of FOXL2 and its mutants in human granulose-like cell lines.

In the present study, it was also found that the expression levels of cell cycle mediators cyclin D1 and CDK4 were reduced and, unexpectedly, CDK6 was increased. Cyclin D1 is reported to be a key element in cell proliferation, whereas CDK4 and CDK6 preferably associate with D-type cyclins during the G1 phase (34). The flow cytometry results indicated that the knockout of FOXL2 restricted cell cycle progression at the G0/G1 phase but did not influence total cell apoptosis. Therefore, these results suggest that the knockout of FOXL2 induced cell cycle arrest. FOXL2 is able to form functional heterodimers with the TGF-â effector transcription factor mothers against decapentaplegic (Smad)3 (35), and an increase in the expression of Smad3 alone can induce the accumulation of cells at G0/G1 arrest through upregulation of the cell cycle progression inhibitors p15/INK4b, p16/INK4a, p19/ARF and p21/CIP1 (36). Therefore, the activity of Smad3 relative protein may require further investigation in FOXL2-knockout cells.

In conclusion, the present study established a stable cell line with disrupted FOXL2 using the CRISPR method in KGN human ovarian granulosa cells. The knockout of FOXL2 restricted cell cycle progression at the G0/G1 phase, but did not influence total cell apoptosis. The expression levels of cell cycle mediators cyclin D1 and CDK4 were reduced, which confirmed that the disruption of FOXL2 may be associated with cell cycle arrest.

\section{Acknowledgements}

The authors would like to thank Professor Yiming $\mathrm{Mu}$ (Chinese PLA General Hospital, Beijing, China) for providing the KGN human ovarian granulosa-like cells.

\section{Funding}

This study was funded by the National Natural Science Foundation of China (grant no. 81471880).

\section{Availability of data and materials}

The datasets used and/or analyzed during the present study are available from the corresponding author on reasonable request.

\section{Authors' contributions}

BT and YZhang performed the experiments, participated in collecting data, and drafted the manuscript. WZ performed the statistical analysis and participated in its design. YZhu and SY contributed to study design, supervised the work, analyzed data and assisted in drafting the manuscript. All authors read and approved the final manuscript. 


\section{Ethics approval and consent to participate}

Not applicable.

\section{Patient consent for publication}

Not applicable.

\section{Competing interests}

The authors declare that they have no competing interests.

\section{References}

1. Crisponi L, Deiana M, Loi A, Chiappe F, Uda M, Amati P, Bisceglia L, Zelante L, Nagaraja R, Porcu S, et al: The putative forkhead transcription factor FOXL2 is mutated in blepharophimosis/ptosis/epicanthus inversus syndrome. Nat Genet 27: 159-166, 2001.

2. Cocquet J, Pailhoux E, Jaubert F, Servel N, Xia X, Pannetier M, De Baere E, Messiaen L, Cotinot C, Fellous M and Veitia RA: Evolution and expression of FOXL2. J Med Genet 39: 916-921, 2002.

3. Loffler KA, Zarkower D and Koopman P: Etiology of ovarian failure in blepharophimosis ptosis epicanthus inversus syndrome: FOXL2 is a conserved, early-acting gene in vertebrate ovarian development. Endocrinology 144: 3237-3243, 2003.

4. Ottolenghi C, Omari S, Garcia-Ortiz JE, Uda M, Crisponi L, Forabosco A, Pilia G and Schlessinger D: Fox12 is required for commitment to ovary differentiation. Hum Mol Genet 14 2053-2062, 2005.

5. Burns KH, Owens GE, Fernandez JM, Nilson JH and Matzuk MM: Characterization of integrin expression in the mouse ovary. Biol Reprod 67: 743-751, 2002.

6. Uhlenhaut NH, Jakob S, Anlag K, Eisenberger T, Sekido R, Kress J, Treier AC, Klugmann C, Klasen C, Holter NI, et al: Somatic sex reprogramming of adult ovaries to testes by FOXL2 ablation. Cell 139: 1130-1142, 2009.

7. Schmidt D, Ovitt CE, Anlag K, Fehsenfeld S, Gredsted L, Treier AC and Treier M: The murine winged-helix transcription factor Fox12 is required for granulosa cell differentiation and ovary maintenance. Development 131: 933-942, 2004

8. Kalfa N, Philibert P, Patte C, Ecochard A, Duvillard P, Baldet P, Jaubert F, Fellous $M$ and Sultan C: Extinction of FOXL2 expression in aggressive ovarian granulosa cell tumors in children. Fertil Steril 87: 896-901, 2007.

9. Moumne L, Batista F, Benayoun BA, Nallathambi J, Fellous M, Sundaresan P and Veitia RA: The mutations and potential targets of the forkhead transcription factor FOXL2. Mol Cell Endocrinol 282: 2-11, 2008.

10. Rosario R, Araki H, Print CG and Shelling AN: The transcriptional targets of mutant FOXL2 in granulosa cell tumours. PLoS One 7: e46270, 2012

11. Horvath P and Barrangou R: CRISPR/Cas, the immune system of bacteria and archaea. Science 327: 167-170, 2010.

12. Terns MP and Terns RM: CRISPR-based adaptive immune systems. Curr Opin Microbiol 14: 321-327, 2011.

13. Wiedenheft B, Sternberg SH and Doudna JA: RNA-guided genetic silencing systems in bacteria and archaea. Nature 482: 331-338, 2012.

14. Hwang WY, Fu Y, Reyon D, Maeder ML, Tsai SQ, Sander JD, Peterson RT, Yeh JR and Joung JK: Efficient genome editing in zebrafish using a CRISPR-Cas system. Nat Biotechnol 31 227-229, 2013

15. Cong L, Ran FA, Cox D, Lin S, Barretto R, Habib N, Hsu PD, Wu X, Jiang W, Marraffini LA and Zhang F: Multiplex genome engineering using CRISPR/Cas systems. Science 339: 819-823, 2013.
16. Wang H, Yang H, Shivalila CS, Dawlaty MM, Cheng AW, Zhang $\mathrm{F}$ and Jaenisch R: One-step generation of mice carrying mutations in multiple genes by CRISPR/Cas-mediated genome engineering. Cell 153: 910-918, 2013.

17. Wang T, Wei JJ, Sabatini DM and Lander ES: Genetic screens in human cells using the CRISPR-Cas9 system. Science 343: 80-84, 2014.

18. Shalem O, Sanjana NE, Hartenian E, Shi X, Scott DA Mikkelson T, Heckl D, Ebert BL, Root DE, Doench JG and Zhang F: Genome-scale CRISPR-Cas9 knockout screening in human cells. Science 343: 84-87, 2014

19. Lee PC, Truong B, Vega-Crespo A, Gilmore WB, Hermann K, Angarita SA, Tang JK, Chang KM, Wininger AE, Lam AK, et al: Restoring ureagenesis in hepatocytes by CRISPR/Cas9-mediated genomic addition to arginase-deficient induced pluripotent stem cells. Mol Ther Nucleic Acids 5: e394, 2016.

20. Tu Z, Yang W, Yan S, Guo X and Li XJ: CRISPR/Cas9: A powerful genetic engineering tool for establishing large animal models of neurodegenerative diseases. Mol Neurodegener 10: 35, 2015.

21. Chang N, Sun C, Gao L, Zhu D, Xu X, Zhu X, Xiong JW and Xi JJ: Genome editing with RNA-guided Cas9 nuclease in zebrafish embryos. Cell Res 23: 465-472, 2013.

22. Yao S, He Z and Chen C: CRISPR/Cas9-mediated genome editing of epigenetic factors for cancer therapy. Hum Gene Ther 26: 463-471, 2015.

23. Gebler C, Lohoff T, Paszkowski-Rogacz M, Mircetic J, Chakraborty D, Camgoz A, Hamann MV, Theis M, Thiede C and Buchholz F: Inactivation of cancer mutations utilizing CRISPR/Cas9. J Natl Cancer Inst 109: pii: djw183, 2016.

24. Kawamura N, Nimura K, Nagano H, Yamaguchi S, Nonomura N and Kaneda Y: CRISPR/Cas9-mediated gene knockout of NANOG and NANOGP8 decreases the malignant potential of prostate cancer cells. Oncotarget 6: 22361-22374, 2015.

25. Livak KJ and Schmittgen TD: Analysis of relative gene expression data using real-time quantitative PCR and the 2(-Delta Delta C(T)) method. Methods 25: 402-408, 2001.

26. Verdin H and De Baere E: FOXL2 impairment in human disease. Horm Res Paediatr 77: 2-11, 2012.

27. Tran S, Zhou X, Lafleur C, Calderon MJ, Ellsworth BS, Kimmins S, Boehm U, Treier M, Boerboom D and Bernard DJ: Impaired fertility and FSH synthesis in gonadotrope-specific Foxl2 knockout mice. Mol Endocrinol 27: 407-421, 2013.

28. Shi F, Ding S, Zhao S, Han M, Zhuang Y, Xu T and Wu X: A piggyBac insertion disrupts Foxl2 expression that mimics BPES syndrome in mice. Hum Mol Genet 23: 3792-3800, 2014.

29. Doudna JA and Charpentier E: Genome editing. The new frontier of genome engineering with CRISPR-Cas9. Science 346: 1258096, 2014

30. Hsu PD, Lander ES and Zhang F: Development and applications of CRISPR-Cas9 for genome engineering. Cell 157: 1262-1278, 2014.

31. Harrison MM, Jenkins BV, O'Connor-Giles KM and Wildonger J: A CRISPR view of development. Genes Dev 28: 1859-1872, 2014.

32. Sander JD and Joung JK: CRISPR-Cas systems for editing, regulating and targeting genomes. Nat Biotechnol 32: 347-355, 2014.

33. Hsu PD, Scott DA, Weinstein JA, Ran FA, Konermann S, Agarwala V, Li Y, Fine EJ, Wu X, Shalem O, et al: DNA targeting specificity of RNA-guided Cas9 nucleases. Nat Biotechnol 31: 827-832, 2013.

34. Asghar U, Witkiewicz AK, Turner NC and Knudsen ES: The history and future of targeting cyclin-dependent kinases in cancer therapy. Nat Rev Drug Discov 14: 130-146, 2015.

35. Blount AL, Schmidt K, Justice NJ, Vale WW, Fischer WH and Bilezikjian LM: FoxL2 and Smad3 coordinately regulate follistatin gene transcription. J Biol Chem 284: 7631-7645, 2009.

36. Huang SM, Lu KT and Wang YC: ATM/ATR and SMAD3 pathways contribute to 3 -indole-induced $\mathrm{G}_{1}$ arrest in cancer cells and xenograft models. Anticancer Res 31: 203-208, 2011. 